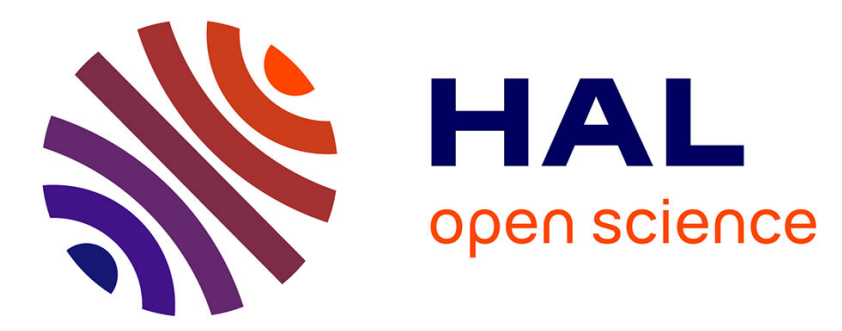

\title{
Identification of crystalline behavior on macroscopic response and local strain field analysis: application to alpha zirconium alloys
}

Lionel Gélébart, Jérôme Crépin, Marie Dexet, Maxime Sauzay, Arjen Roos

\section{- To cite this version:}

Lionel Gélébart, Jérôme Crépin, Marie Dexet, Maxime Sauzay, Arjen Roos. Identification of crystalline behavior on macroscopic response and local strain field analysis: application to alpha zirconium alloys. Journal of ASTM International (JAI), 2004, 1, 10.1520/JAI12333 . hal-00111436

\author{
HAL Id: hal-00111436 \\ https://hal.science/hal-00111436
}

Submitted on 4 Sep 2019

HAL is a multi-disciplinary open access archive for the deposit and dissemination of scientific research documents, whether they are published or not. The documents may come from teaching and research institutions in France or abroad, or from public or private research centers.
L'archive ouverte pluridisciplinaire HAL, est destinée au dépôt et à la diffusion de documents scientifiques de niveau recherche, publiés ou non, émanant des établissements d'enseignement et de recherche français ou étrangers, des laboratoires publics ou privés.

\section{(ㅇ)(1) $\$$}

Distributed under a Creative Commons Attribution - NonCommerciall 4.0 International 
L. Gélébart, ${ }^{1}$ J. Crépin, ${ }^{2}$ M. Dexet, ${ }^{3}$ M. Sauzay, ${ }^{4}$ and A. Roos $^{5}$

\title{
Identification of Crystalline Behavior on Macroscopic Response and Local Strain Field Analysis: Application to Alpha Zirconium Alloys
}

\begin{abstract}
The purpose of this paper is to present an identification method of the crystalline behavior of a material from a mechanical test performed on a polycrystalline sample. Because of the lack of knowledge about its crystalline behavior, this method is applied to a Zirconium alloy. This identification is based on a finite element modeling of the microstructure, and the results are compared to both the macroscopic and the microscopic experimental results. On the microscopic scale, the plastic strains are obtained using a micro-extensometry technique, and the crystalline orientation using an EBSD technique. In order to validate the method, an identification is performed with only two free parameters: the evolutions of the macroscopic and microscopic errors appear to be regular and exhibit a well-defined minimum so that the parameters can be clearly identified.
\end{abstract}

KEYWORDS: identification, crystalline plasticity, zirconium, finite-element, micro-extensometry, EBSD

\section{Introduction}

On different scales, the recent development of displacement (strain) field measurement techniques opens new possibilities for the identification of the mechanical behavior of materials.

From a practical point of view, in case of a "classical" macroscopic identification, they can be used to control the homogeneity of the strain field at the surface of the specimen. But their main interest is that this condition of homogeneity is no more a necessity: actually, the displacement, and consequently the strain, are known in a high number of points (typically about 10 000), so that an identification of the materials' behavior based on "heterogeneous" tests can be considered [1]. An application can also be performed at the scale of the microstructure, i.e., the grain for a polycrystal, thanks to strain field measurements (micro-extensometry) and orientation field measurements (EBSD) $[2,3]$. On this scale, the purpose is to identify the crystalline behavior of the grain.

At least two different methods can be considered to use these fields for the identification procedure. On the one hand, it is possible to perform finite-element calculations and to compare the experimental and simulated strain fields. In this case, the evaluated stress field is equilibrated, and the quantity to minimize is the difference between experimental and simulated

\footnotetext{
${ }^{1}$ Research Engineer, CEA Saclay /DEN/DMN/SRMA, lionel.gelebart@cea.fr.

${ }^{2}$ Research Engineer, LMS Ecole Polytechnique, crepin@lms.polytecnique.fr.

${ }^{3}$ Ph.D. Student, CEA Saclay/DEN/DMN/SRMA, marie.dexet@cea.fr, and LMS Ecole Polytechnique, dexet@1ms.polytechnique.fr.

${ }^{4}$ Research Engineer, CEA Saclay /DEN/DMN/SRMA, maxime.sauzay@cea.fr.

${ }^{5}$ Research Engineer, ONERA, Arjen.Roos@onera.fr.
} 
strain fields $[2,3]$. On the other hand, it is possible to use the experimental strain field to evaluate a stress field through a direct integration of the mechanical behavior's constitutive law. In this case, the stress field is no more equilibrated, and the quantity to minimize is now the equilibrium gap evaluated on this stress field [1].

The crystalline behavior of $\alpha-\mathrm{Zr}$ alloys is still a problem for the micro-mechanical modeling of fuel cladding in the nuclear industry; for example, the critical resolved shear stresses of the four types of slip systems are difficult to identify. One reason for this difficulty is the strong heterogeneity of the stress field due to the strong anisotropy of the plastic behavior. One way to take it into account is to perform finite-element calculations. The work presented below is then carried out on the scale of the microstructure, and the purpose is the identification of the $\alpha-\mathrm{Zr}$ crystalline behavior from tests performed on poly-crystal specimens. The aim is to use both the macroscopic response and the intra-granular strain field to identify the parameters of the crystalline behavior.

The identification is applied to a tensile test performed on a $\mathrm{Zr}$ alloy, and the outline of this paper follows the different points of this methodology: first, the experimental procedure leading to the superposition of the experimental plastic strain and crystalline orientation fields, then, the finite element simulation, next, the definition of an error between experimental and simulated results obtained on the local and on the macroscopic scale, and finally, a way to use this error to identify crystalline parameters.

\section{Material}

The tensile test specimen (Fig. 1) is taken from a rolled and recrystallized plate $(8 \mathrm{~mm}$ in thickness) with the tensile test direction parallel to the transverse direction (perpendicular to the rolling direction and to the normal of the plate). The material is the so-called grade $702 \mathrm{Zr}$ alloy (chemistry is given Table 1). The strong texture of this alloy (Fig. 2) is typical of rolled and recrystallized $\alpha-Z r$ alloys. The average grain size is $12 \mu \mathrm{m}$.

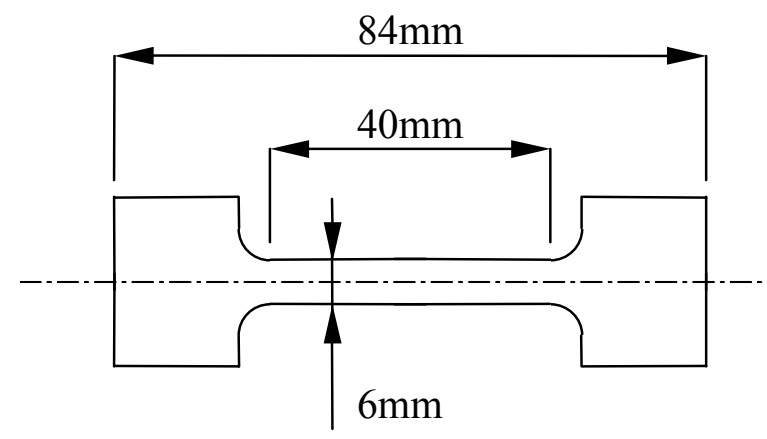

FIG. 1-Geometry of the tensile test specimen (3mm in thickness).

TABLE 1-Chemical composition of zirconium grade 702.

\begin{tabular}{ccccccccc}
\hline Element & $\mathrm{C}$ & $\mathrm{H}$ & $\mathrm{O}$ & $\mathrm{N}$ & $\mathrm{Cr}$ & $\mathrm{Fe}$ & $\mathrm{Ni}$ & $\mathrm{Sn}$ \\
\hline $\begin{array}{c}\text { Concentration } \\
\text { (ppm weight) }\end{array}$ & 58 & $4-7$ & $\begin{array}{l}1,40 \\
0\end{array}$ & 33 & 240 & 760 & 50 & $\begin{array}{l}2,28 \\
0\end{array}$ \\
\hline
\end{tabular}



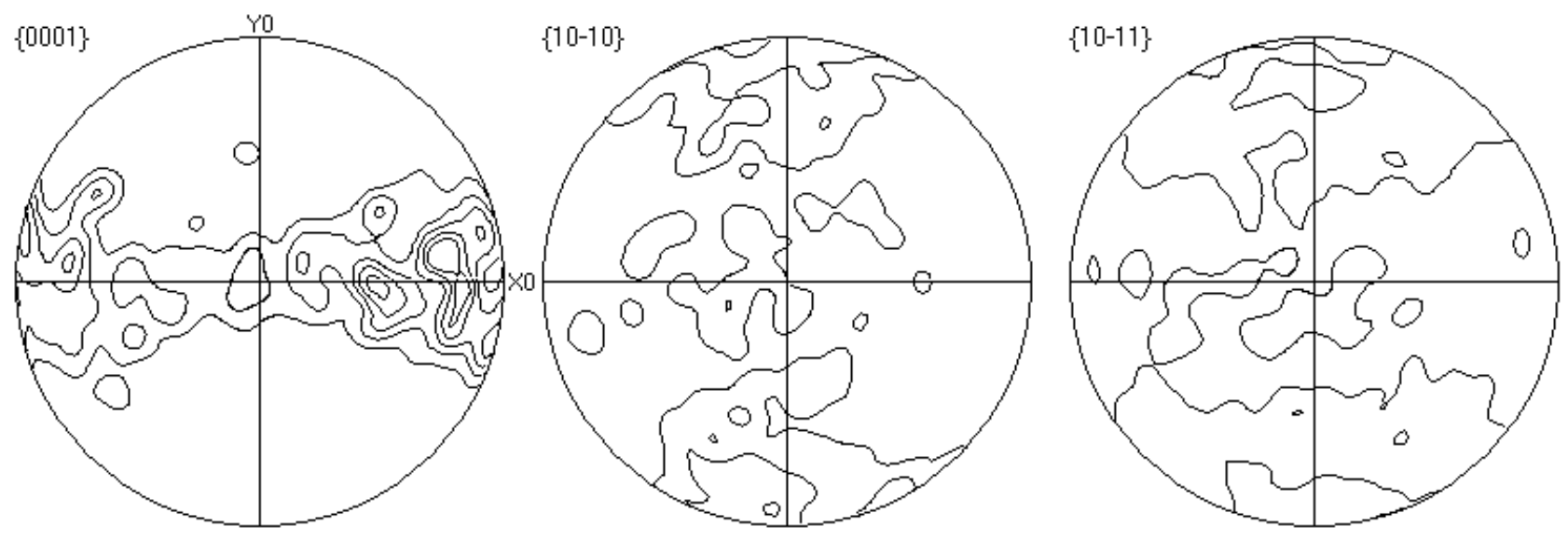

FIG. 2-Crystallographic texture ( $D L=$ longitudinal direction, and $D T=$ transverse direction).

\section{Experiments}

In this part, we present the tensile test that gives the macroscopic behavior of the material, the measurement of the crystallographic orientation field that will be used to define a finite element model of the microstructure, and the measurement of the plastic strain field that will be compared to the finite element results.

\section{Tensile Test}

The tensile test specimen was deformed at a uniform temperature of $200^{\circ} \mathrm{C}\left( \pm 2^{\circ} \mathrm{C}\right)$ with a crosshead displacement rate of $1 \mathrm{~mm} / \mathrm{min}$ that corresponds to an approximate strain rate of $4.10^{-4}$ $\mathrm{s}^{-1}$. The axial strain was measured by means of an axial extensometer. The corresponding macroscopic stress-strain curve is given Fig. 3. Two constraints have determined the choice of the temperature: it had to be low enough to preserve the quality of the micro-grids (a gold deposit) and high enough to avoid twinning that is hard to take into account in the modeling of the plastic crystalline behavior.

\section{Orientation Field Measurement (EBSD)}

The lattice orientation of each grain is obtained by EBSD, whose electron beam displacement can be prescribed automatically by using a regular mesh $(1 \mu \mathrm{m}$ step) to get the whole orientation field (Fig. 4) associated with the studied microstructure on a surface $\left(150 \times 150 \mu \mathrm{m}^{2}\right)$.

\section{Superimposition}

In Figs. 4 and 6, four marks made of a $10 \times 10 \mu \mathrm{m}^{2}$ gold deposit at the surface of the specimen surrounding the analysis region are illustrated. They are used to superimpose the crystallographic orientation map (Fig. 4), obtained for a tilt angle of $70^{\circ}$ and the secondary electron (SE) images (Fig. 6), obtained with no tilt of the specimen.

Actually, because of inherent alignment, imperfections between the tilt axis and the scanning direction, an image obtained with a tilt of $70^{\circ}$ is "deformed" compared to the image obtained with no tilt; the effective scanned zone is not rectangular (Fig. 5). 


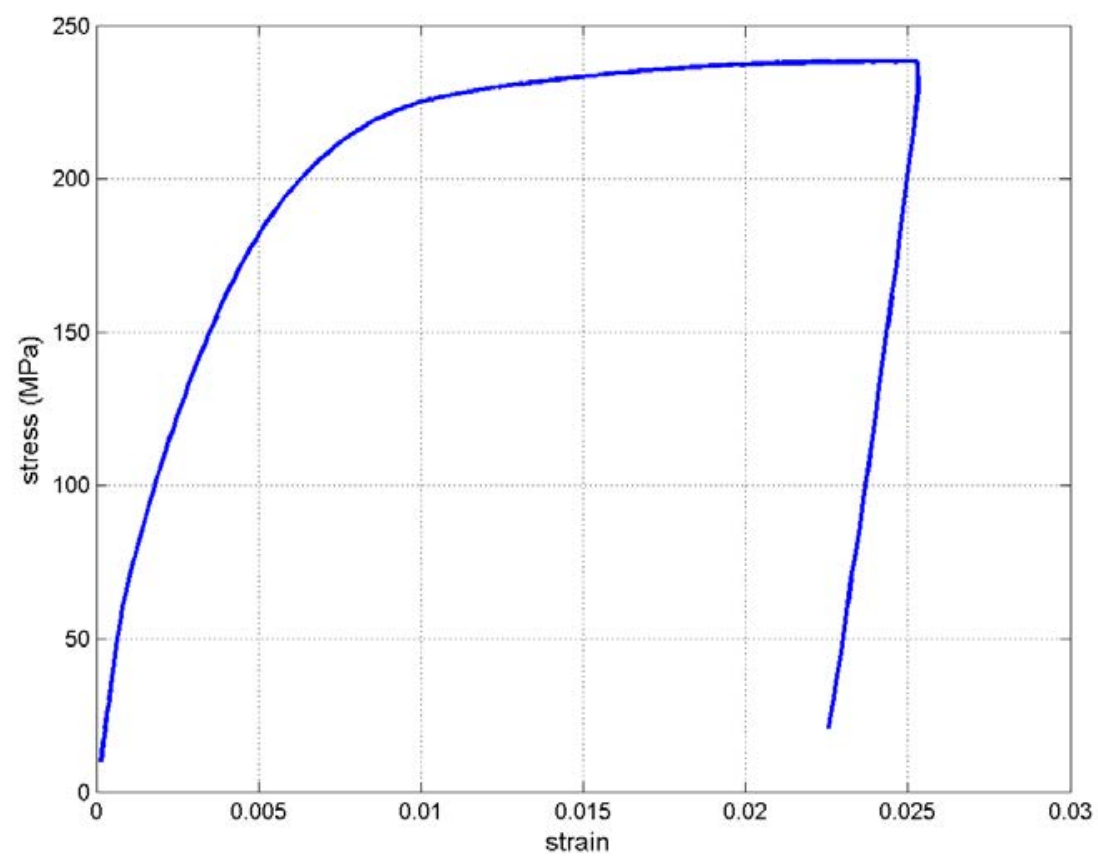

FIG. 3-Macroscopic behavior.

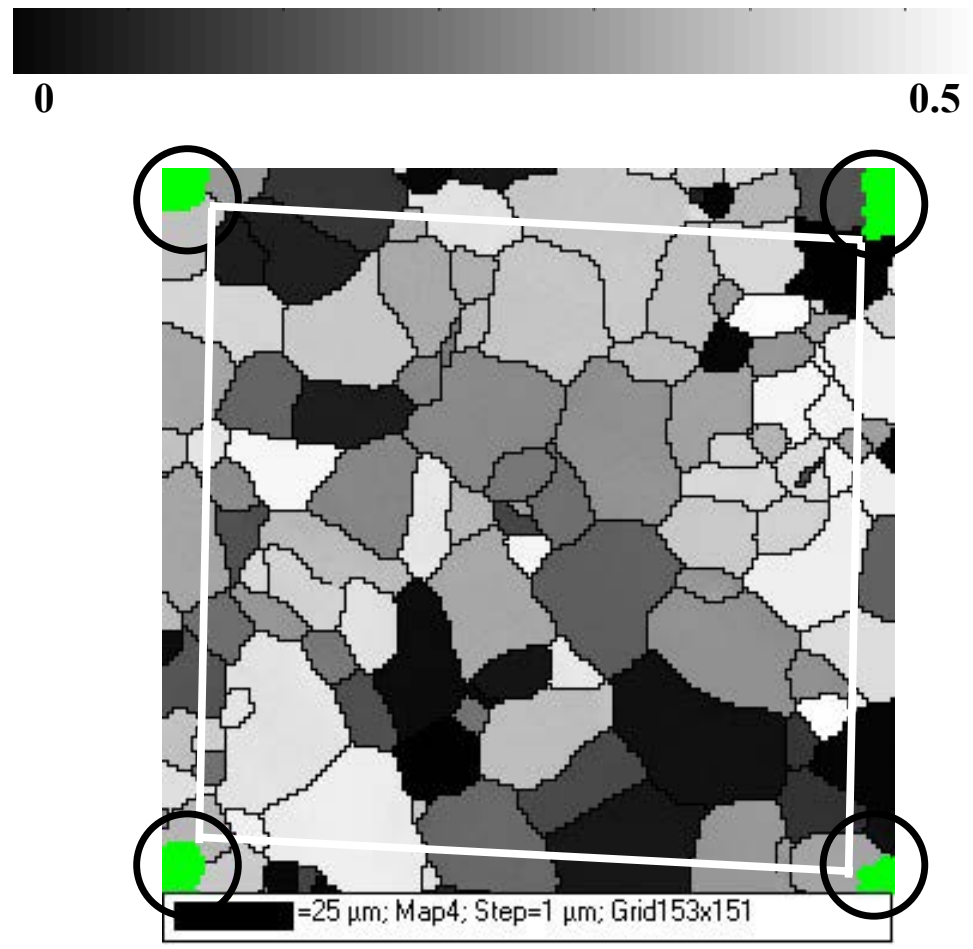

FIG. 4-Schmid factor for prismatic slip. 


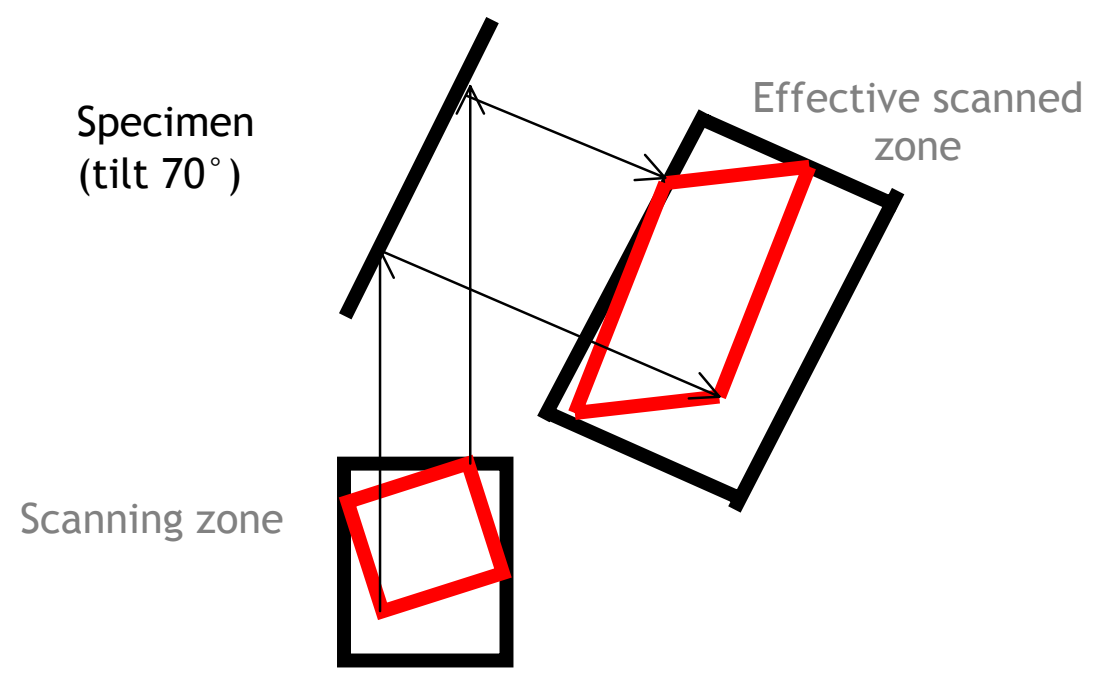

FIG. 5-Distortion of the EBSD image.

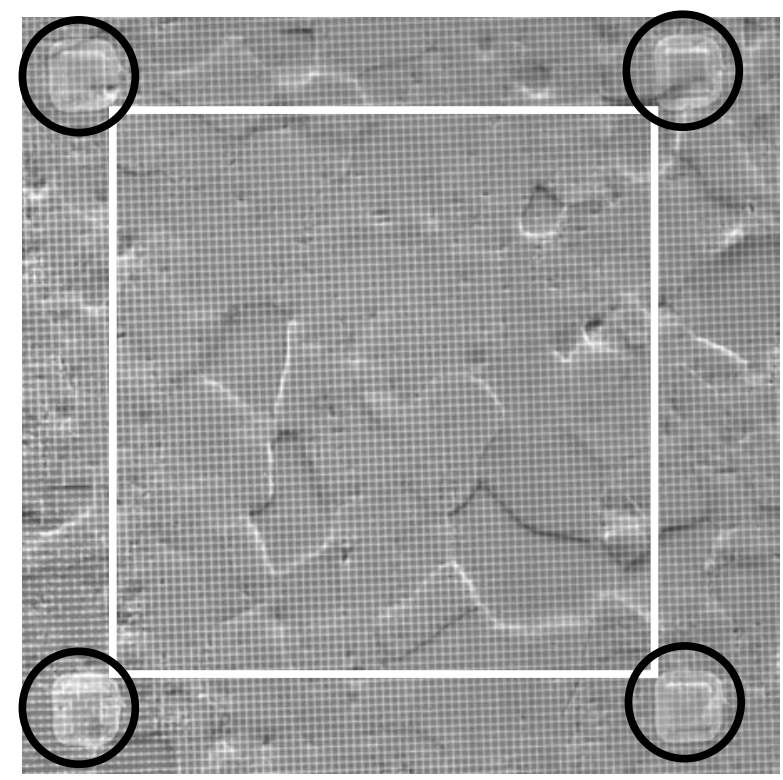

FIG. 6-SE image: the micro-grid used for strain field measurement and the marks $(10 \times 10$ $\left.\mu \mathrm{m}^{2}\right)$ used for the superposition.

In the context of a quantitative analysis, an accurate superposition of the orientation field on the SE images, used to evaluate the displacement field, is required: a local strain must not be affected to a wrong grain. The problem of the superposition is to determine the transformation $\Phi_{\mathrm{c}}$ between $\underline{X}$, the coordinates of a point in the EBSD image, and $\underline{x}$, the coordinate of the same point in the SE image. If this transformation is assumed to be homogeneous, the knowledge of this transformation in three points is enough to determine its six components; this is performed thanks to the gold marks (Figs. 4 and 6). 


\section{Displacement and Strain Field Measurement (Micro-Extensometry)}

The local strain field at the surface of the sample is obtained by a micro-extensometry technique, developed by LMS-Ecole Polytechnique [4,5], whose principle consists in taking SEM images of a square micro-grid deposited on the surface, before and after deformation (or during deformation if the test is performed in situ). The in-plane displacements of the intersections of this micro-grid are determined by means of a correlation method that compares the images before and after deformation. The strain field is then derived from this displacement field. The choice of the pitch for the micro-grids, $2 \mu \mathrm{m}$, is related to the accuracy and resolution required to measure intra-granular strain fields. The strain measured after unloading with this technique is the total residual strain field; it is divided into the plastic and the residual elastic strain fields, and it is assumed that the residual elastic local strains can be neglected in front of the plastic strains.

The superimposition of the equivalent plastic strain map on the microstructure can be observed in Fig. 7 where the microstructure is represented by the Schmid factor's map for prismatic slip. The strain distribution is very heterogeneous and can be described by bands of plastic strain localization, having an orientation of around $45^{\circ}$ with respect to the tensile axis, and regions remaining nearly undeformed. These regions correspond to grains where the Schmid factor for prismatic slip is low, and there are often localization bands situated at the boundaries of these grains, especially if these boundaries have an orientation of $45^{\circ}$ with respect to the tensile axis.
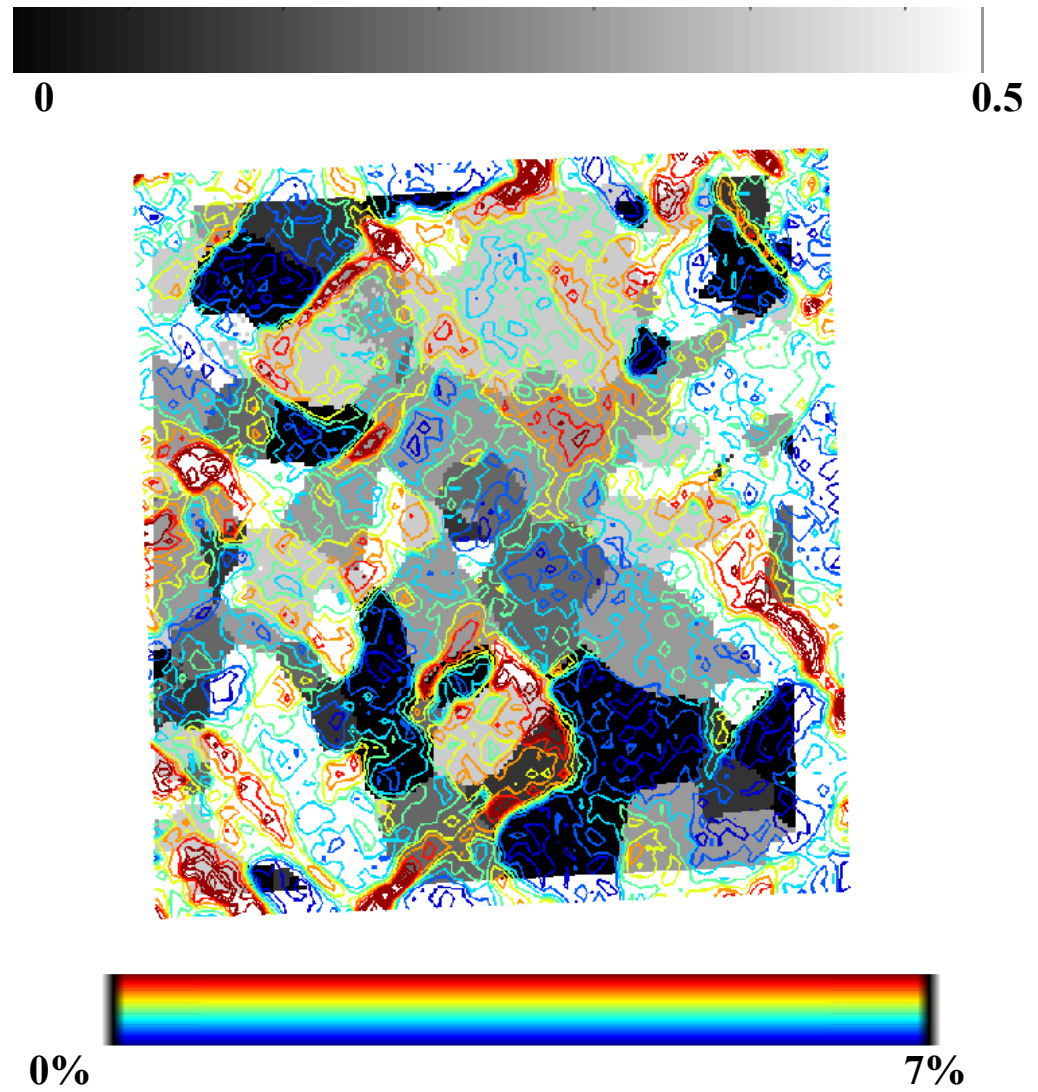

FIG. 7-Superposition of the experimental plastic strain map ( $\left.\varepsilon_{\text {eq exp }}\right)$ on the Schmid factor's map (for prismatic slip); the tensile test axis is horizontal. 


\section{Crystalline Behavior}

In this section we present the crystalline behavior law that will be, in the next sections, associated to the crystallographic orientation field in the finite element model.

\section{Deformation Mechanisms [7,8]}

The major deformation mode is prismatic glide on $\{10 \overline{10}\}$ planes with Burgers vectors $<\mathrm{a}>=<11 \overline{2} 0>$. The main secondary slip system is pyramidal $<\mathrm{a}>$ slip gliding on $\{1 \overline{101}\}$ planes, but pyramidal $<\mathrm{c}+\mathrm{a}>(<\overline{2} 113>\{1101\})$ and basal slip $(<11 \overline{2} 0>\{0001\})$ also have been observed. At low temperature, twinning also plays an important role in the plastic deformation. The critical resolved shear stresses and the interactions between the different systems still are not well known.

\section{Crystalline Behavior Law}

As a first approximation, we use a simple and classical single crystal behavior law written in the context of small perturbations. The elastic behavior is given by the Hooke law (Eq 1), $\underline{\underline{\sigma}}$ being the stress, $\underline{\underline{\varepsilon}}$ the total strain, and $\underline{\varepsilon}^{v p}$ the visco-plastic strain.

$$
\underline{\underline{\sigma}}=K:\left(\underline{\varepsilon}-\underline{\underline{\varepsilon}}^{v p}\right)
$$

The elastic tensor $K$ has a transverse isotropic symmetry, and its numerical value is given as a function of the temperature in [6]. According to the normality rule, the visco-plastic strain rate is the sum of the shear strain rates on all the slip systems (s) (Eqs 2 and 3).

$$
\begin{aligned}
& \underline{\dot{\varepsilon}}^{v p}=\sum_{s} \dot{\gamma}_{s}^{v p} \underline{\underline{R}}_{s} \\
& \underline{\underline{\underline{R}}} s=\frac{1}{2}\left(\underline{n}_{s} \otimes \underline{m}_{s}\right)^{s y m}
\end{aligned}
$$

$\dot{\gamma}_{s}^{v p}$ is the intensity of the shear strain rate, and $\underline{\underline{R}}_{s}$ gives the orientation of the shear strain on the system (s) defined by its gliding plane and direction, respectively $\underline{n}_{s}$ and $\underline{m}_{s}$. On each system, the intensity of the shear strain rate is related to the applied stress by a modified Norton law (Eqs 4 and 5). The introduction of a sinh function was proposed for zirconium alloys [8-11].

$$
\begin{aligned}
& \dot{\gamma}_{s}^{v p}=\dot{\gamma}_{0}\left(\sinh \left(\frac{\left|\tau_{s}\right|}{\tau_{s}^{0}}\right)\right)^{n} \operatorname{sign}\left(\tau_{s}\right) \\
& \tau_{s}=\underline{\underline{\sigma}}: \underline{\underline{R_{s}}}
\end{aligned}
$$


Where $\tau_{s}$ and $\tau_{s}^{0}$ are the resolved and critical resolved shear stresses on the system $(s)$. Finally the evolution of the critical resolved shear stress (i.e., the hardening behavior) is assumed to be linear (Eq 6).

$$
\dot{\tau}_{s}^{0}=\sum_{k} h_{s k}\left|\dot{\gamma}_{k}^{v p}\right|
$$

The hardening matrix, a $24 \times 24$ matrix, has been simplified (Eqs 7 and 8). It can be described by only two values, $h_{0}$ for the self-hardening, and $h_{0} q$ for the latent hardening.

$$
\begin{aligned}
& h_{s k}=h_{0} q_{s k} \\
& q_{s k}=q+(1-q) \delta_{s k}
\end{aligned}
$$

\section{Finite Element Calculation with Experimental Boundary Conditions}

The geometry of the finite element mesh is built on the description of the EBSD mesh; it consists of one layer of cubic elements (8 nodes - 8 Gauss points), the center of each element being a position of the EBSD mesh. For each element, the material behavior is given by the crystalline behavior of the hexagonal crystal, described previously, associated to the crystallographic orientation (EBSD).

On the upper and lower faces, boundary conditions consist of conditions of free surface so that this calculation is close to a plane stress calculation. These conditions assume that the effect of the underlying microstructure can be neglected if we only consider the strains at the surface of the sample, but this question still has to be clarified.

The boundary conditions applied to the contour of the mesh are divided into an elastic and a plastic part (Eqs 9-11). In Eqs 9-13, $U_{X X X}$ are displacement fields restricted to the points located at the contour.

$$
\begin{aligned}
& U(t)=U_{E}(t)+U_{P}(t) \\
& U_{E}(t)=f(t) U_{E \max } \\
& U_{P}(t)=g(t) U_{P \max }
\end{aligned}
$$

The plastic part at the end of the loading $U_{P \max }$ is assumed to be equal to the experimental displacement field at the contour (Eq 12) measured after loading, thanks to the micro-grid (as there is not an exact coincidence between the points of the FEM mesh and those of the microgrid, a linear interpolation is applied to get the displacement at the points of the contour).

$$
U_{P \max }=U_{\exp }
$$


The elastic part at the end of the loading $U_{E \max }$ is assumed to be equal to the displacement field at the contour corresponding to a homogeneous strain $E_{0}(\mathrm{Eq} 13) . E_{0}$ is evaluated as the strain that gives $\left\langle\sigma_{x x}\right\rangle=\sigma_{\exp }^{\max },\left\langle\sigma_{y y}\right\rangle=0$ and $\left\langle\sigma_{x y}\right\rangle=0$ if the behavior of the material is assumed to remain elastic.

$$
U_{E \max }=\left\{\underline{u}(\underline{x})=\underline{\underline{E_{0}}} \underline{\underline{x}}, \underline{x} \in \text { contour with } \underline{\underline{E_{0}}} /\left\langle\sigma_{x x}\right\rangle=\sigma_{\exp }^{\max },\left\langle\sigma_{y y}\right\rangle=0,\left\langle\sigma_{x y}\right\rangle=0 \text { in elasticity }\right\}
$$

The kinetic of the loading is given by the functions $\mathrm{f}$ and $\mathrm{g}$. The most simple to apply is a proportional loading $\left(f(t)=g(t)=\frac{t}{t_{f}}\right)[2,3]$, but it is not quite realistic; at the beginning of the tensile test, the material is elastic, and $\left\langle\varepsilon_{y y}\right\rangle /\left\langle\varepsilon_{x x}\right\rangle \approx-0.37$, and at the end its behavior is plastic, and $\left\langle\varepsilon_{y y}\right\rangle /\left\langle\varepsilon_{x x}\right\rangle \approx-0.75$ (this value is due to the anisotropy of the material; it would have been equal to -0.5 for an isotropic material). A way to improve the description of the loading is to define a piecewise evolution (Fig. 8), with $\sigma_{\text {exp }}^{y}$ and $t_{y}$ the stress and the time at the beginning of plasticity (Fig. 9).

The use of experimental boundary conditions represents the interaction with the other grains situated in the neighborhood of the area of interest.

The finite element code used for these calculations is CASTEM2000 developed by CEASaclay/SEMT [12], and the crystalline behavior [13] was introduced in the code by CEASaclay/SRMA.

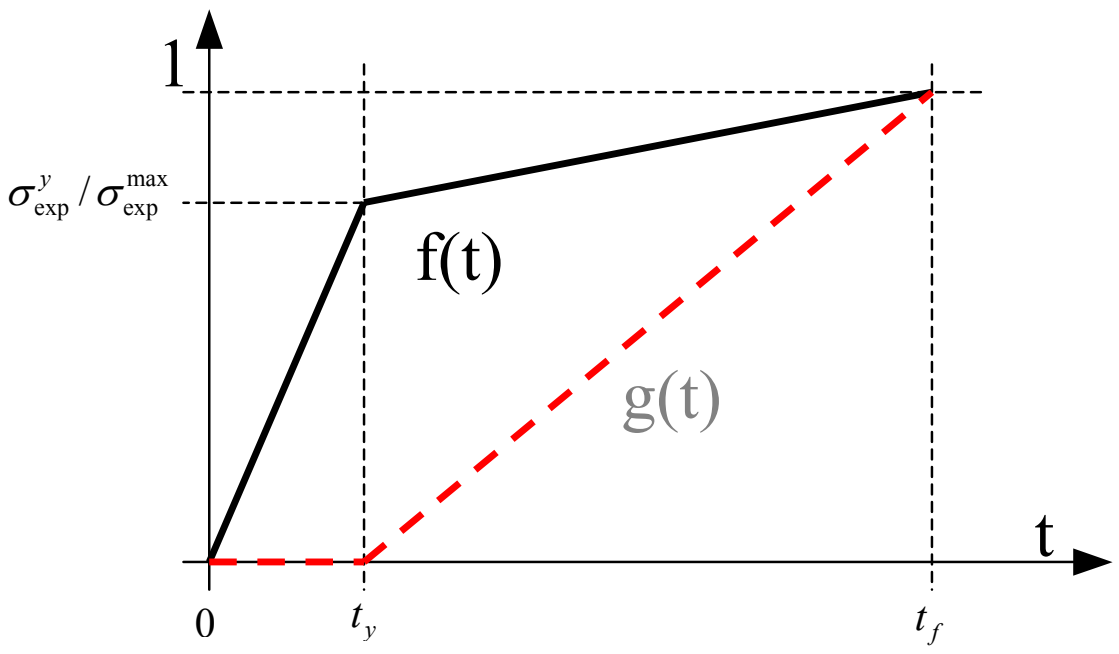

FIG. 8- Evolution of the functions that define the evolution of the boundary conditions. 


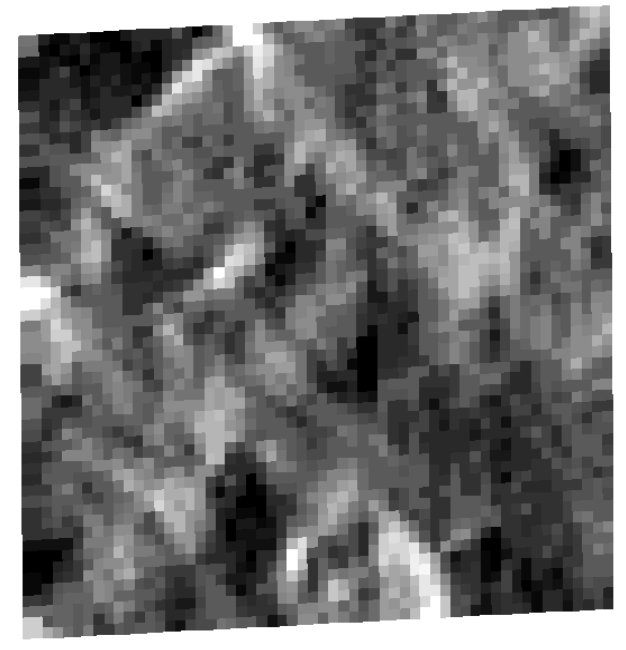

(a)
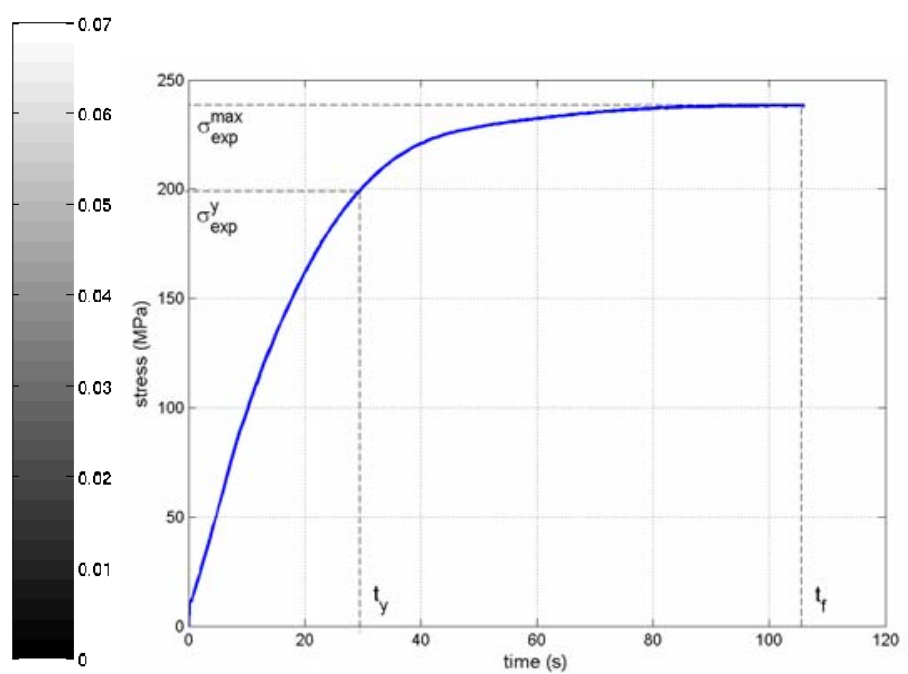

(b)

FIG. 9-Experimental plastic strain ( $\varepsilon_{x x}^{P}$ ) (a) and experimental macroscopic stress (b) $\left\langle\varepsilon_{x x}^{P}\right\rangle=2.6 \%$.

\section{Definition of the Error Estimation}

In this section, we define the error estimation that quantifies the "quality" of the finite element calculation compared to the experimental results, and as a consequence, the "quality" of the set of parameters used for this calculation. As usual in identification problems, many different choices can be made to define the error estimation; here is one choice.

The error estimation is divided into two parts: a macroscopic and a microscopic one. The macroscopic error (Eq 14) is the norm between the mean simulated stress tensor and the experimental macroscopic stress tensor (with $\sigma_{y y \exp }=\sigma_{x y \exp }=0$ for a uniaxial tensile test), averaged during the time of loading. Of course, the larger and the more representative the region of interest is, the more the use of this macroscopic error makes sense.

$$
E^{m a c}=\frac{1}{t_{f}} \int_{0}^{t_{f}}\left\|\langle\underline{\underline{\underline{\sigma}}}\rangle-\underline{\underline{\sigma_{\exp }}}\right\|_{2} d t \text { where }\|\underline{\underline{A}}\|_{2}=\sqrt{\underline{\underline{A}}: \underline{\underline{A}}}=\sqrt{A_{x x}^{2}+A_{y y}^{2}+2 A_{x y}^{2}}
$$

The microscopic error used in reference [2] for body-centered cubic (BCC) crystals was based on the comparison between the statistical distributions of experimental and simulated strains. This definition can be used in BCC structures because all the slip systems have the same critical resolved shear stress. In our case, the mechanisms have different CRSS, and the positions of the strain heterogeneities have to be taken into account: the microscopic error (Eq 15) is the average of the norm of the difference between the simulated plastic strain tensor and the experimental strain tensor. The choice of the norm $\|\cdot\|_{e q}$ is done because it only takes into account the deviatoric part of the tensor, which is the important part if we are interested in plastic deformation. 


$$
E^{m i c}=\left\langle\left\|\varepsilon^{P}-\varepsilon_{\exp }\right\|_{e q}\right\rangle \quad \text { where }\|\underline{\underline{A}}\|_{e q}=\frac{2}{3} \sqrt{\left(A_{x x}-A_{y y}\right)^{2}+4 A_{x y}^{2}}
$$

In order to define a single error estimation, these errors have to be normalized by macroscopic and microscopic normalization factors (Eq 16). The choice that has been made for this normalization is to use the errors ( $E_{0}^{m a c}$ and $E_{0}^{m i c}$ ) evaluated with a uniform and isotropic behavior (i.e., a Von Mises yield function). With this choice of normalization, the error estimation indicates the "quality" of a set of crystalline parameters in comparison with an isotropic behavior. Details will be found in the applications.

$$
E=\frac{1}{2}\left(\frac{E^{m a c}}{E_{0}^{m a c}}+\frac{E^{m i c}}{E_{0}^{m i c}}\right)
$$

\section{Applications}

The experimental results that will be used for the following applications are: the evolution of the experimental axial stress as a function of time (Fig. 9b) and the experimental plastic strain field (Fig $9 a$ ) on a region of $116 \times 110 \mu \mathrm{m}^{2}$. With this size of region, the finite element mesh has 3190 elements, and the calculation lasts around one hour on an Intel Xeon 3.06GHz processor.

\section{Effect of the Boundary Conditions Applied on the Contour}

In order to evaluate quantitatively the effect of the use of experimental boundary conditions (BC) as they were described in a previous section, another calculation was performed assuming homogeneous $\mathrm{BC}$ : the plastic part of the displacement is assumed to be equal to the displacements corresponding to an homogeneous strain $\underline{\underline{E_{0}^{P}}}$, where $\underline{\underline{E_{0}^{P}}}$ is equal to the average experimental plastic strain $\left(E_{0 x x}^{P}=2.6 \% ; E_{0 y y}^{P}=-2.1 \% ; E_{0 x y}^{P}=0.2 \%\right)(\mathrm{Eq} 17)$.

$$
U_{P \max }=\left\{\underline{u}(\underline{x})=\underline{\underline{E_{0}^{P}}} \underline{\underline{x}}, \underline{x} \in \partial S \quad \text { with } \quad \underline{\underline{E_{0}^{P}}}=\left\langle\underline{\underline{\varepsilon_{\exp }}}\right\rangle\right\}
$$

A part of the material parameters used for this comparison is inspired from literature; they have been identified to fit creep tests thanks to a micro-mechanical model [10]. The other parameters have been qualitatively identified on the macroscopic behavior, keeping in mind the experimental observations on the relative ease of slip of the different slip systems (Table 2).

TABLE 2-Choice of material parameters.

\begin{tabular}{lccccc}
\hline & $\dot{\gamma}_{0} *\left(\mathrm{~s}^{-1}\right)$ & $n^{*}$ & $\tau^{O}(\mathrm{MPa})$ & $h_{0}(\mathrm{MPa})$ & $q^{*}$ \\
\hline Prismatic & $1.10^{7}$ & 3 & 20 & 100 & 2 \\
Pyramidal $<\mathrm{a}>$ & $1.10^{7}$ & 7 & 40 & 100 & 2 \\
Basal & $1.10^{7}$ & 7 & 80 & 100 & 2 \\
Pyramidal $<\mathrm{c}+\mathrm{a}>$ & $1.10^{7}$ & 7 & 80 & 100 & 2 \\
\hline
\end{tabular}

$*$ inspired from [10]. 
A qualitative comparison (Fig. 10) shows that the plastic strain field is more heterogeneous when it is evaluated with experimental BC. From a quantitative point of view, the microscopic errors $E^{\text {mic }}$ are of $1.54 \%$ and $1.74 \%$ for experimental and homogeneous conditions; the first one leads to a best estimate of the experimental plastic strain field.

Another way to evaluate the effect of the experimental BC is to perform a calculation with a homogeneous and isotropic behavior. The behavior is elasto-plastic with an isotropic Von Mises yield function and a linear hardening. A value of $100 \mathrm{MPa}$ is chosen for the linear hardening, and the yield stress is identified on the axial stress at the end of the loading. The plastic strain field (Fig. 11) is also heterogeneous with localization bands, but the heterogeneities are more diffuse in comparison with the heterogeneous crystalline calculation (Fig. 10a), and surprisingly, the microscopic error $E_{0}^{\text {mic }}$, equal to $1.37 \%$, is lower. Not surprisingly, from a macroscopic point of view (Fig. 11b), the evolution of the axial stress is far from the experimental one and the transversal stress is far from 0.MPa that corresponds to a uniaxial tensile test. The macroscopic error $E_{0}^{m a c}$ is then of $99.3 \mathrm{MPa}$. This example reveals the necessity to take both the microscopic and the macroscopic behavior into account to identify the material behavior.

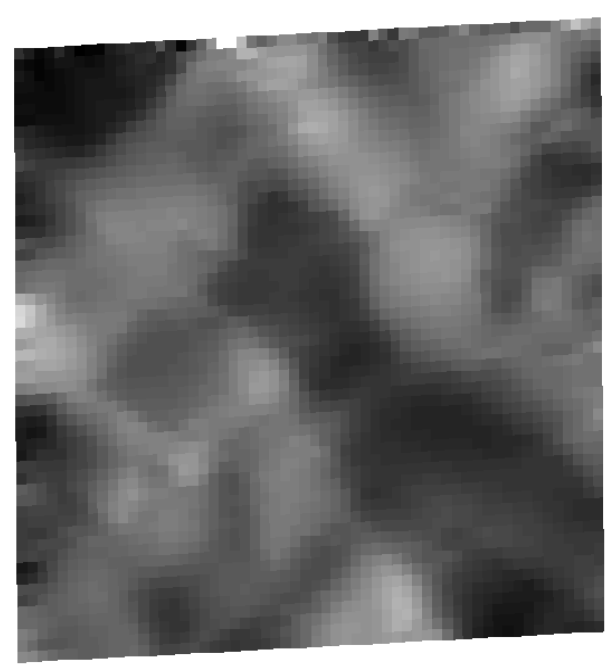

(a)

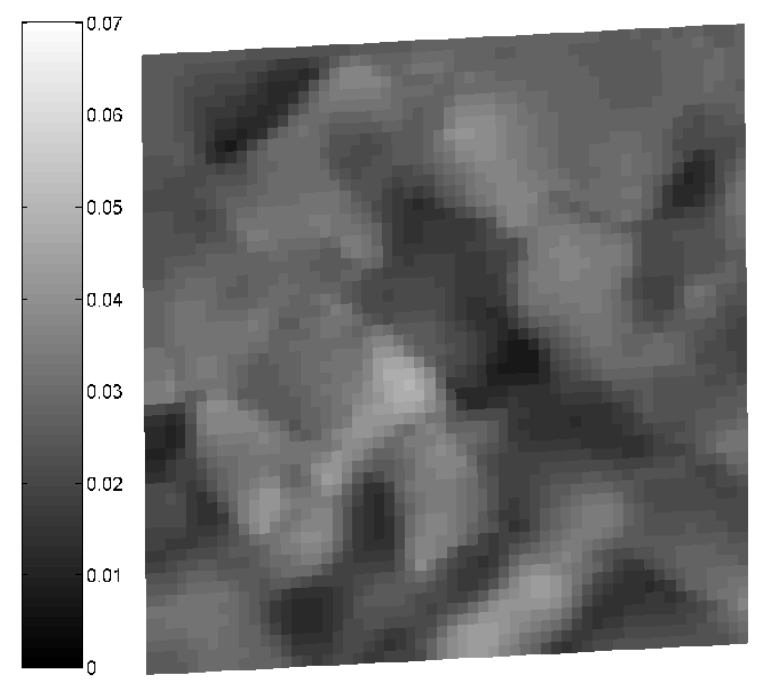

(b)

FIG. 10 - Comparison of the plastic strain field $\left(\varepsilon_{x x}^{P}\right)$ calculated with experimental boundary conditions (a) or homogeneous boundary conditions (b). 


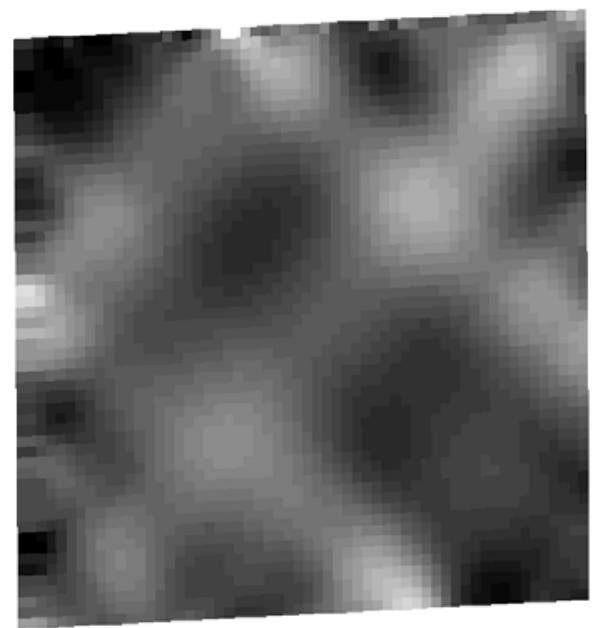

(a)
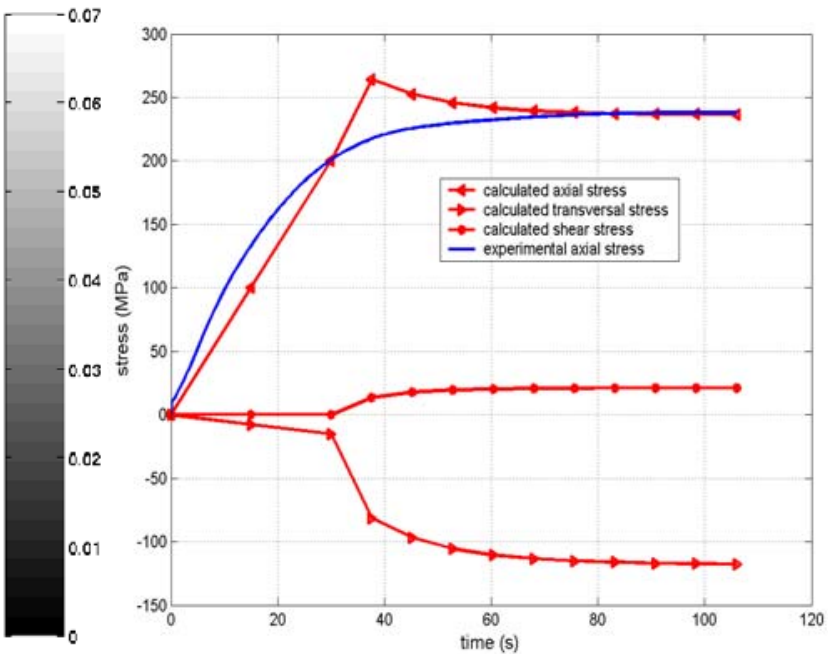

(b)

FIG. 11-Plastic strain field $\left(\varepsilon_{x x}^{P}\right)$ and evolution of the macroscopic stresses for a homogeneous and isotropic behavior (Von Mises).

\section{Identification of Material Parameters}

General Description-From the description of the crystalline behavior, the material parameters are $\left(\tau_{P}^{0}, \tau_{\pi<a>}^{0}, \tau_{B}^{0}, \tau_{\pi<c+a>}^{0}, \ldots\right)$. A way to identify these parameters is to apply directly a minimization procedure to the error estimation $E$ as a function of $\left(\tau_{P}^{0}, \tau_{\pi<a>}^{0}, \tau_{B}^{0}, \tau_{\pi<c+a>}^{0}, \ldots\right)$.

Here, we propose to modify the description of the problem and to use the parameters $\left(\tau_{P}^{0}, \alpha_{\pi<a>}, \alpha_{B}, \alpha_{\pi<c+a>}, \ldots\right)$, where $\alpha_{i}=\frac{\tau_{i}^{0}}{\tau_{P}^{O}}$. Now, a pre-identification of $\tau_{P}^{0}$ can be performed easily; it consists in identifying $\tau_{P}^{0}$ on the axial stress at the end of the loading, the other parameters being constant. As a consequence the error estimation, $E$ is now a function of $\left(\alpha_{\pi<a>}, \alpha_{B}, \alpha_{\pi<c+a>}, \ldots\right)$; for each evaluation of $E\left(\alpha_{\pi<a>}, \alpha_{B}, \alpha_{\pi<c+a>}, \ldots\right)$ an optimal value of $\tau_{P}^{0}$ is identified. The main advantage of this pre-identification is that all the evaluations of $E$ have in common a macroscopic behavior "not so different from" the experimental one. The second advantage is that this pre-identification has a very low cost in terms of iterations.

The use of a minimization procedure has not been carried out yet, and as it is important to have a preliminary knowledge of the behavior of the function $E$ before using such a procedure, the following example is restricted to the identification of two parameters.

Example: Identification of $\tau_{P}^{0}$ and $\alpha_{\pi<a>}$-In this example, the material parameters are those used for the comparison between experimental and homogeneous boundary conditions (Table 2), except the critical resolved shear stresses (CRSS): $\tau_{P}^{0}$ and $\alpha_{\pi<a>}$ are still to identify, $\alpha_{B}$ and $\alpha_{\pi<c+a>}$ are set equal to 4 . The criterion $\left(\sigma_{x x}\left(t_{f}\right)-\sigma_{x x \exp }\left(t_{f}\right) / \sigma_{x x \exp }\left(t_{f}\right)\right)$ used for the preidentification of $\alpha_{\pi<c+a>}$ is $1 \%$, and the number of iterations to reach this criterion varies between 2 and 4 . As the CRSS of the pyramidal $<$ a $>$ slip is supposed to be larger than the CRSS 
of prismatic slip and lower than the CRSS of the basal and pyramidal $<\mathrm{c}+\mathrm{a}>$ slips, the parameter $\alpha_{\pi<a>}$ is bounded by the values 1 and 4 .

The evolutions of the microscopic and macroscopic errors $\left(E_{0}^{m i c}\right.$ and $\left.E_{0}^{m a c}\right)$ as functions of $\alpha_{\pi<a>}$ (Fig. 12a), are quite regular, and they both exhibit the presence of minima that correspond respectively to the interpolated values of 2.8 and 2.3 . The error estimation $E$ that mixes the microscopic and the macroscopic errors (Eq 16) exhibit a unique minimum for $\alpha_{\pi<a>}=2.6$ (Fig. $12 b)$. Each point on Fig. 12 is the result of the pre-identification, and the pre-identified value of $\tau_{P}^{0}$ is also represented; it decreases when $\alpha_{\pi<a>}$ increases, which is not surprising. The corresponding optimal set of CRSS is given in Table 3. Finally, the macroscopic curves and the plastic strain fields are presented (Fig. 13) for three values of $\alpha_{\pi<a>}$, the extreme values 1 and 4 and the value 2.5 corresponding to the minimum of $E$.

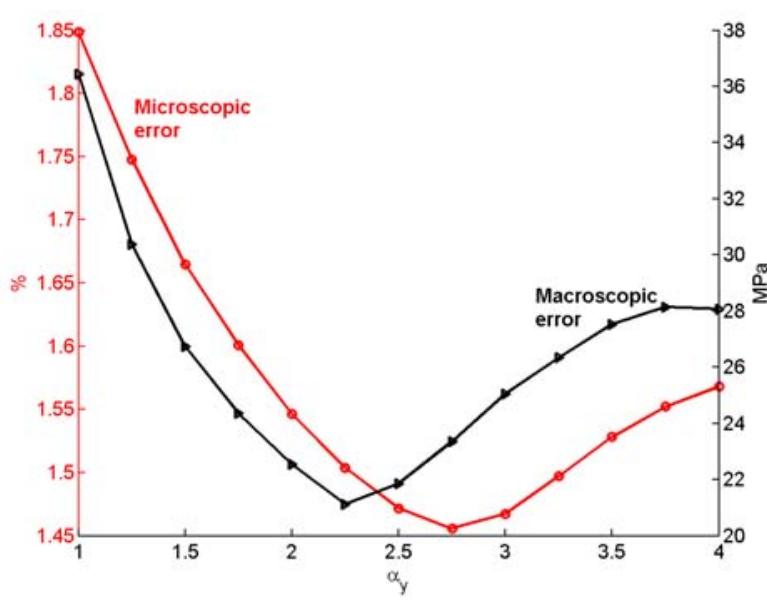

(a)

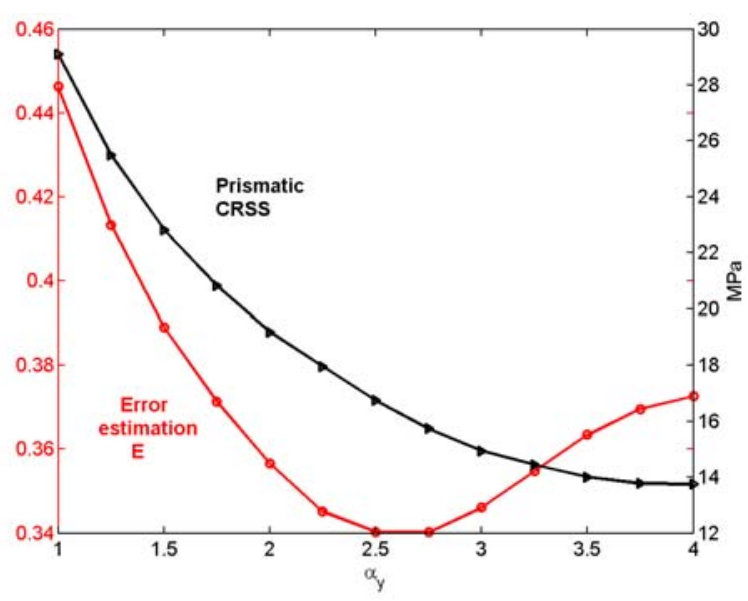

(b)

FIG. 12-Evolutions of the microscopic and macroscopic errors (a), of the error estimation (b) and of the pre-identified CRSS for prismatic slip (b).

TABLE 3-Optimal values of prismatic and pyramidal $<a>C R S S$, obtained with $\alpha_{B}=\alpha_{P y<c+a>}=4$.

\begin{tabular}{lcc}
\hline & Prismatic & Pyramidal $<\mathrm{a}>$ \\
\hline$\alpha$ & 1. & 2.6 \\
CRSS $(\mathrm{MPa})$ & 16.2 & 42.1 \\
\hline
\end{tabular}




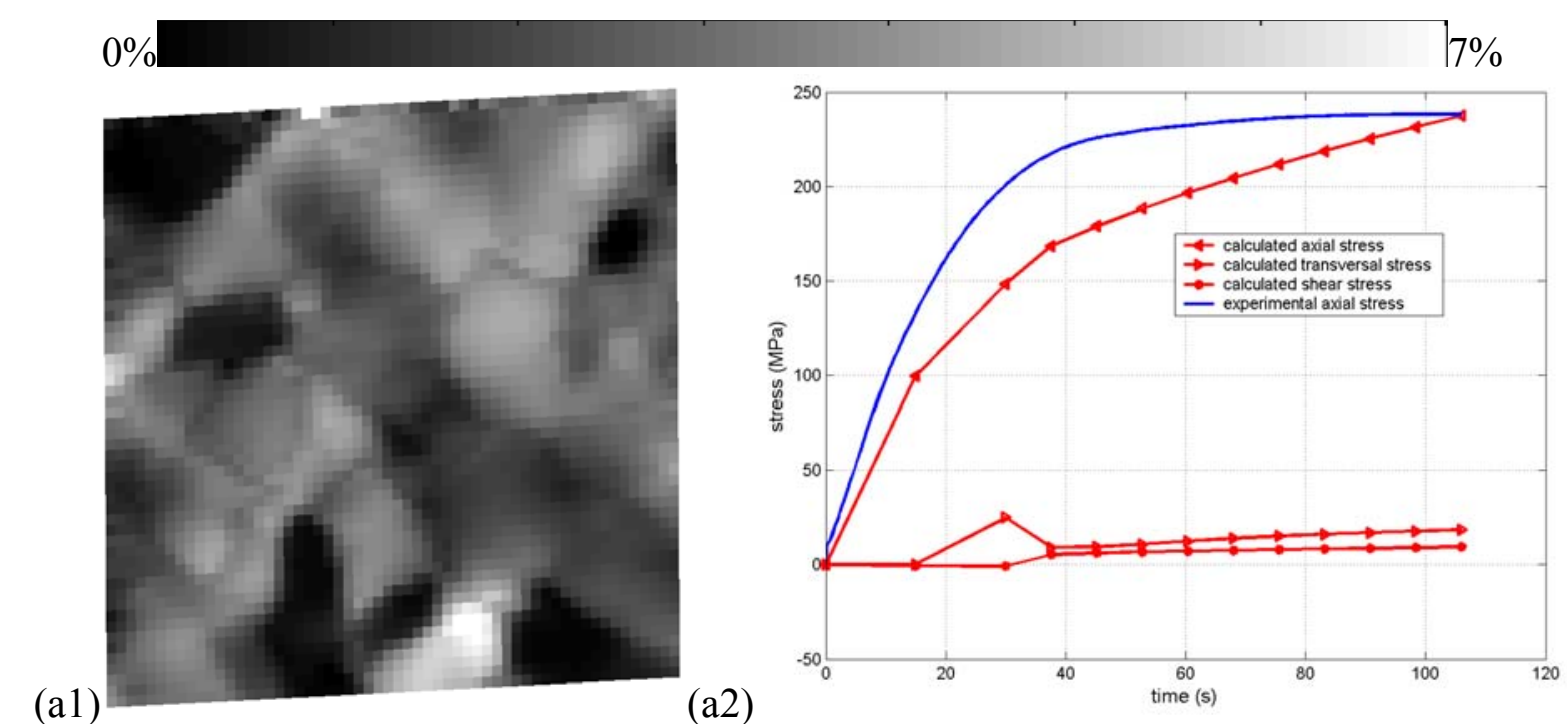

(a1)

(a2)

(b1)

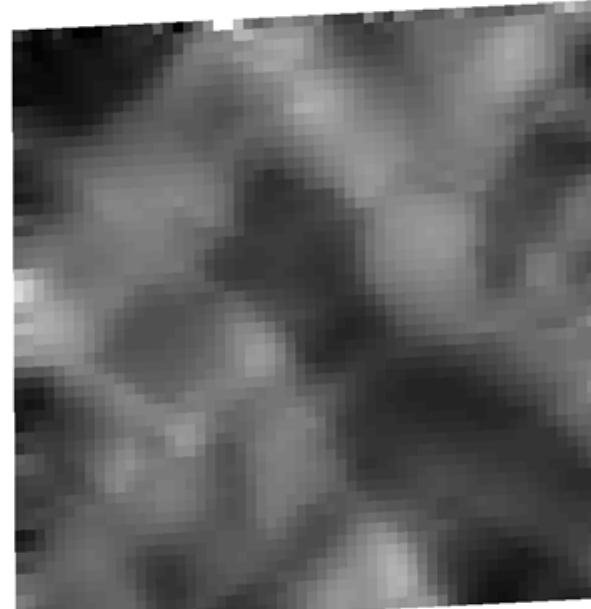

(b2)

(c1)

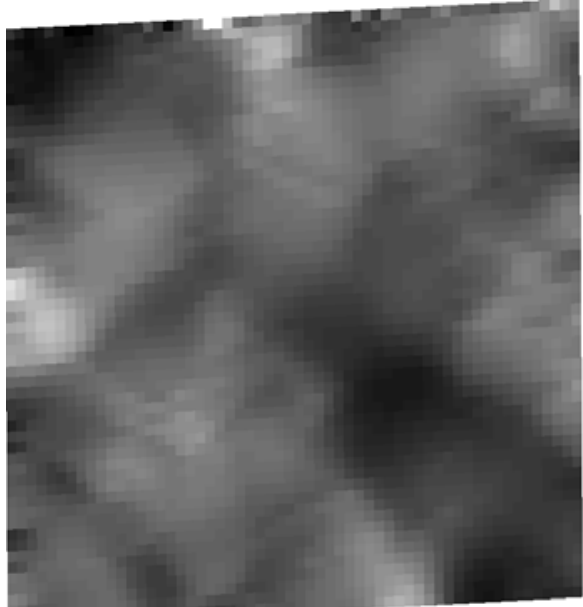

(c2)

FIG. 13-Plastic strain field $\left(\varepsilon_{x x}^{P}\right)$ and macroscopic behavior for $\alpha_{\pi<a>}=1$ (a1 and a2), $\alpha_{\pi<a>}=2.5$ (b1 and b2), and $\alpha_{\pi<a>}=4$ (c1 and $\mathrm{c} 2$ ). 


\section{Conclusion, Discussion, and Future Prospects}

The aim of this paper was to present an identification method of the crystalline behavior of the $\alpha-Z r$ alloy grain from a mechanical test performed on a polycrystalline sample. Actually, in many cases, single crystals having an identical chemical composition to that of the alloy are hard to obtain. Then, the transposition of the material parameters obtained on a single crystal to the behavior of the grain is not evident. As a consequence, this behavior has to be identified on the polycrystalline material.

A way to identify this local behavior is to use a micromechanical model and to fit this behavior on the macroscopic response obtained on the polycrystal [14]. But in this approach, the local quantities such as stress and strains, on which the micromechanical models are based, are not compared to the experimental local quantities. The purpose of this approach is to perform this identification not only on the macroscopic behavior but also on the local quantities.

The local quantity that we use is the plastic strain field, measured on the scale of the microstructure due to a micro-extensometry technique. The micromechanical model is a finiteelement calculation with a crystalline behavior associated to a crystallographic orientation measurement technique (EBSD). In order to take into account the interaction of the grains in the neighborhood of the region of interest, experimental boundary conditions are used. The macroscopic error accounts for all of the components of the stress tensor. The microscopic error accounts for the difference, at each point, between the experimental and the simulated plastic strain tensor.

A first identification has been proposed in order to validate this methodology: only two parameters were free. The evolutions of the microscopic and macroscopic errors appear to be regular and exhibit a well-defined minimum so that the parameters can be identified clearly. Such identifications still have to be performed before using a minimization procedure for a more complex identification.

The crystalline behavior, which is quite simple and classical, will have to be modified in order to take physical aspects of the plastic deformation into account, such as the evolutions of the dislocation densities. Finally, two questions, on which this identification method is based, are still open and will have to be clarified: is the region of interest mechanically representative of the polycrystal behavior, and what is the effect of the microstructure under the surface?

\section{References}

[1] Claire, D., Hild, F., and Roux, S., "Identification of Damage Fields Using Kinematic Measurements," CRAS Mécanique, 330, 2002, pp. 729-734.

[2] Hoc, T., Crépin, J., Gélébart, L., and Zaoui, A., "A Procedure for Identifying the Plastic Behavior of Single Crystals from the Local Response of Polycrystals," Acta Materialia, 51/18, 2004, pp. 5479-5490.

[3] Gélébart, L., "Approche multi-échelles du comportement mécanique de l'alliage Ti$\mathrm{Al}_{48} \mathrm{Cr}_{2} \mathrm{Nb}_{2}$, Ph.D. thesis, Ecole Polytechnique, France, 2002.

[4] Allais, L., Bornert, M., Bretheau, T., and Caldemaison, D., "Experimental Characterization of the Local Strain Field in a Heterogeneous Elastoplastic Material," Acta Materialia, Vol. 42, No. 11, 1994, pp. 3865-3880. 
[5] Soppa, E., Doumalin, P., Binkele, P., Wiesendanger, T., Bornert, M., and Schmauder, S., "Experimental and Numerical Characterisation of In-Plane Deformation in Two-Phase Materials," Computational Materials Science, Vol. 21, No. 3, 2001, pp. 261-275.

[6] Northwood, D. O., London, I. M., Bahen, L. E., "Elastic Constants of Zr Alloys,” Journal of Nuclear Materials, Vol. 55, No. 3, 1975, pp. 299-310.

[7] Tenckhoff, E., "Deformation Mechanisms, Texture, and Anisotropy in Zirconium and Zircaloy," ASTM STP 966, ASTM International, West Conshohocken, PA.

[8] Geyer, P., "Comportement élasto-viscoplastique de tubes en Zircaloy-4," Ph.D. thesis, Ecole Nationale Supérieure des Mines de Parise, France, 1999.

[9] Masson R., "Estimations non-linéaires du comportement global de matériaux hétérogènes en formulation affine - Application aux alliages de Zironium," Ph.D. thesis, Ecole Polytechnique, France, 1998.

[10] Brenner, R., Bechade, J. L., Castelnau, O., and Bacroix, B., "Thermal Creep of Zr--Nb1\%-O Alloys: Experimental Analysis and Micromechanical Modeling," Journal of Nuclear Materials, Vol. 305, Nos. 2-3, 2002, pp. 175-186.

[11] Brenner, R., "Influence de la microstructure sur le comportement en fluage thermique d'alliages de zirconium: analyse expérimentale et mise en œuvre de méthodes d'homogénéisation," Ph.D. thesis, Université Paris XIII, France, 2001.

[12] Cast3M available on http://www-cast3m.cea.fr.

[13] Héraud, S., "Du polycristal au multicristal: élaboration d'un mésoscope numérique pour une analyse locale en élastoviscoplasticité," Ph.D. thesis, Ecole Polytechnique, France, 1998.

[14] Tomé, C. N., So, C. B., Woo, C. H., "Self-Consistent Calculation of Steady-State Creep and Growth in Textured Zirconium," Phil. Mag. A, 67, 1993, pp. 917-930. 